\title{
15. PRELIMINARY DATING BY FOSSIL CALCAREOUS NANNOPLANKTON, DEEP SEA DRILLING PROJECT, LEG 8
}

\author{
W. W. Hay, Rosenstiel School of Marine and Atmospheric Sciences, University of Miami, \\ Miami, Florida
}

\section{INTRODUCTION}

The zonation used here is that of Boudreaux and Hay (1969) for the Pleistocene, Martini and Worsley (1970) for the Neogene, and Hay et al. (1967), Bramlette and Wilcoxon (1967) and Hay (1970) for the Paleogene.

All samples were examined, although in most instances time permitted only a cursory inspection for this preliminary determination.

\section{HOLE 69}

Sample 69-1-1, $37 \mathrm{~cm}$ :

Discoaster argutus and a few poor coccoliths present. Age: Probably Middle Miocene, but exact age assignment impossible.

Sample 69-2-1, 30-31 cm:

Very few, poorly preserved nannofossils; no age assignment possible.

Sample 69-2-2, 6-7 cm:

Discoaster argutus, $D$. cf. exilis?.

Age: Probably Middle Miocene.

Sample 69-2-3, 6-7 cm:

Indeterminate Discoaster spp. present.

Sample 69-2-4, 6-7 cm:

Barren.

Sample 69-2-5, 6-7 cm:

Discoaster argutus, D. kugleri, D. cf. brouweri, Coccolithus pelagicus.

Age: D. kugleri Zone (Middle Miocene).

Sample 69-2-6, 6-7 cm:

Discoaster brouweri, D. extensus, D. deflandrei?, $D$. kugleri, coccolith rims.

Age: D. kugleri Zone (Middle Miocene).

Sample 69-3-1, 6-7 cm:

Sample 69-3-2, 6-7 cm:

Discoaster brouweri, D. extensus, D. kugleri, poorly preserved coccoliths.

Sample 69-3-3, 6-7 cm:

Barren.
Sample 69-3-4, 6-7 cm:

Very poor, but like Sample 3-2, 6-7 cm.

Sample 69-3-5, 6-7 cm:

Sample 69-3-6, 6-7 cm:

Discoaster druggii, D. nephados, D. obtusus, very few coccoliths.

Age: Probably Sphenolithus belemnos Zone (Early Miocene).

Sample 69-4-1, 7-8 cm:

Sample 69-4-2, 6-7 cm:

Sample 69-4-3, 3-4 cm:

Sample 69-4-4, 6-7 cm:

Sample 69-4-5, 6-7 cm:

Sample 69-4-6, 6-7 cm:

Triquetrorhabdulus carinatus, Discoaster nephados, $D$. trinidadensis, Sphenolithus belemnos.

Age: $T$. carinatus Zone (Early Miocene).

Sample 69-5-1, 6-7 cm:

Sample 69-5-2, 6-7 cm:

Reticulofenestra umbilica, $R$. bisecta, Cyclococcolithus floridanus, many coccoliths.

Age: Early Oligocene.

Sample 69-5-3, 6-7 cm:

Sample 69-5-4, 6-7 cm:

As above, but very poor assemblage.

Sample 69-5-5, 6-7 cm:

Discoaster tani ornatus, Reticulofenestra umbilica, $R$. bisecta, Cyclococcolithus floridanus, many coccoliths. Age: Early Oligocene.

Sample 69-6-1, 6-7 cm: Sample 69-6-2, 6-7 cm: Sample 69-6-3, 6-7 cm: Sample 69-6-4, 6-7 cm: Sample 69-6-5, 6-7 cm: Sample 69-6-6, 6-7 cm: Barren.

\section{HOLE 69A}

Sample 69A-1-1, 4-5 cm:

Sample 69A-1-2, 6-7 cm:

Sample 69A-1-3, 6-7 cm:

Sample 69A-1-4, 6-7 cm: 
Sample 69A-1-5, 6-7 cm:

Sample 69A-1-6, 6-7 cm:

Triquetrorhabdulus carinatus, Discoaster nephados, $D$. trinidadensis, $D$. cf. druggii, many coccoliths.

Age: Lowermost $D$. druggii Zone or uppermost $T$. carinatus Zone (Early Miocene). Assemblages poorly preserved.

Sample 69A-2-1, 6-7 cm:

Very poor nannofossils.

Sample 69A-2-2, 6-7 cm:

Sample 69A-2-3, 6-7 cm:

Sample 69A-2-4, 6-7 cm:

Sample 69A-2-5, 6-7 cm:

Sample 69A-2-6, 6-7 cm:

Discoaster nephados, D. trinidadensis, D. dilatus,

Triquetrorhabdulus carinatus.

Age: T. carinatus Zone (Early Miocene).

Sample 69A-3-1, 63-64 cm:

Sample 69A-3-2, 6-7 cm:

Sample 69A-3-3, 6-7 cm:

Sample 69A-3-4, 6-7 cm:

Sample 69A-3-5, 6-7 cm:

Sample 69A-4-1, 6-7 cm:

Sample 69A-4-2, 6-7 cm:

Sample 69A-4-3, Top:

Sample 69A-4-4, Top cm:

Sample 69A-4-5, 6-7 cm:

Sample 69A-4-6, 6-7 cm:

Sample 69A-5-1, 6-7 cm:

Discoaster nephados, D. trinidadensis, $D$. cf. deflandrei, few coccoliths.

Age: Late Oligocene.

Sample 69A-5-2, 6-7 cm:

Very poor assemblage.

Sample 69A-5-3, 6-7 cm:

Sample 69A-5-4, 6-7 cm:

Sample 69A-5-5, 6-7 cm:

Sample 69A-6-1, 6-7 cm:

Sample 69A-6-2, 6-7 cm:

Discoaster nephados, $D$. cf. deflandrei, Reticulofenestra bisecta, but generally poor assemblages.

Age: Late Oligocene.

Sample 69A-6-3, 6-7 cm:

As above, but with Sphenolithus ciperoensis.

Age: Probably Sphenolithus distentus Zone (Late Oligocene).

Sample 69A-6-5, Top:

Very poor assemblage.

Sample 69A-7-1, 30-31 cm:

Discoaster nephados, $D$. cf. deflandrei, $D$. saundersi.
Age: Probably Sphenolithus distentus or S. predistentus Zone (Late or Middle Oligocene).

Sample 69A-7-2, 30-31 cm:

Flood of Sphenolithus pacificus.

Sample 69A-7-3, 71-72 cm:

Very poor assemblage.

Sample 69A-8-1, 6-7 cm:

Poor assemblage with Discoaster spp., Reticulofenestra umbilica, and a few other poorly preserved coccoliths. Age: Oligocene.

Sample 69A-8-2, 6-7 cm:

Sample 69A-8-3, 112-113 cm:

Very poor assemblages.

Sample 69A-8-4, $52-53 \mathrm{~cm}$ :

Poor assemblage like Sample 8-1, 6-7 cm.

Sample 69A-8-5, 6-7 cm:

Very poor assemblage.

Sample 69A-9-1, 6-7 cm:

Sample 69A-9-2, 6-7 cm:

Sample 69A-9-3, 6-7 cm:

Sample 69A-9-4, 6-7 cm:

Sample 69A-9-5, 6-7 cm:

Barren.

Sample 69A-10-1, 6-7 cm:

Very poor assemblage with only a few coccoliths present.

Sample 69A-10-2, 6-7 cm:

Reticulofenestra umbilica, $R$. bisecta, Discoaster cf. deflandrei.

Age: Probably Early Oligocene.

Sample 69A-10-3, 6-7 cm:

Sample 69A-10-4, 6-7 cm:

Sample 69A-10-5, 6-7 cm:

Sample 69A-10-6, 20-21 cm:

Sample 69A-11-1, 6-7 cm:

Sample 69A-11-2, 16-17 cm:

Sample 69A-11-3, 6-7 cm:

Sample 69A-11-4, 6-7 cm:

Sample 69A-11-5, 6-7 cm:

Sample 69A-11-6, 6-7 cm:

Barren.

Sample 69A-12-1, Top at 20:

Discoaster deflandrei, D. cf. tani, flood of Ericsonia subdisticha and many other small coccoliths.

Age: Probably Ericsonia subdisticha Zone (Early Oligocene). 


\section{HOLE 70}

Sample 70-1-4, 6-7 cm:

Sample 70-2-1, 33-34 cm:

Barren.

Sample 70-2-2, 33-34 cm:

Discoaster challengeri, D. variabilis, D. extensus, D. brouweri, D. quinqueramus, few coccoliths.

Age: Mixed Early Pliocene and Late Miocene.

Sample 70-3-1, 6-7 cm:

Sample 70-3-2, 6-7 cm:

Barren.

Sample 70-3-3, 6-7 cm:

Poorly preserved assemblage with indeterminate asteroliths and coccoliths.

Sample 70-3-4, 6-7 cm:

Sample 70-3-5, 6-7 cm:

Discoaster argulus, D. dilatus, poorly preserved coccoliths.

Age: Probably Middle or Early Miocene.

Sample 70-4-1, 6-7 cm:

Sample 70-4-2, 12-13 cm:

Sample 70-4-3, 6-7 cm:

Sample 70-4-4, 14-15 cm:

Sample 70-4-4, 8-9 cm:

Discoaster trinidadensis, D. cf. deflandrei, D. cf. saundersi, D. obtusus, poorly preserved.

Age: Early Miocene, probably Sphenolithus belemnos Zone.

Sample 70-5-1, 7-8 cm:

Sample 70-5-2, 16-17 cm:

Discoaster trinidadensis, D. deflandrei, D. argutus, D. dilatus, D. extensus.

Age: Probably Sphenolithus belemnos Zone (Early Miocene).

Sample 70-5-3, 6-7 cm:

Sample 70-5-4, 6-7 cm:

Discoaster extensus, D. dilatus, D. nephados, D. cf. druggii.

Age: Probably Sphenolithus belemnos Zone (Early Miocene).

Sample 70-5-5, 6-7 cm:

Sample 70-5-6, 6-7 cm:

Barren.

Sample 70-6-1, 6-7 cm: Sample 70-6-2, 6-7 cm: Sample 70-6-3, 6-7 cm: Sample 70-6-4, 6-7 cm: Sample 70-6-5, 6-7 cm:
Sample 70-6-6, 6-7 cm:

Sample 70-7-1, 6-7 cm:

Sample 70-7-2, 6-7 cm:

Sample 70-7-3, 6-7 cm:

Sample 70-7-4, 6-7 cm:

Discoaster druggii, D. nephados, D. dilatus, D. argutus, D. obtusus.

Age: Sphenolithus belemnos Zone (Early Miocene).

Sample 70-7-5, 6-7 cm:

Sample 70-7-6, 6-7 cm:

Sample 70-8-1, 6-7 cm:

Sample 70-8-2, 6-7 cm:

Sample 70-8-3, 6-7 cm:

Sample 70-8-4, 6-7 cm:

Sample 70-8-5, 6-7 cm:

Sample 70-8-6, 6-7 cm:

Sample 70-9-4, Bottom:

Sample 70-9-5, 6-7 cm:

Sample 70-9-6, 6-7 cm:

Sample 70-9-6, 100-110 cm:

Sample 70-10-3, 6-7 cm:

Sample 70-10-4, 6-7 cm:

Sample 70-10-5, 6-7 cm:

Sample 70-10-6, 6-7 cm:

Triquetrorhabdulus carinatus, Discoaster druggii, $D$. nephados, D. dilatus, D. argutus, D. obtusus.

Age: D. druggii Zone (Early Miocene).

Sample 70-11-5, 6-7 cm:

Sample 70-11-5, 63-64 cm:

Sample 70-11-6, 6-7 cm:

Sample 70-12-1, Bottom:

Sample 70-12-2, 6-7 cm:

Sample 70-12-4, 6-7 cm:

Sample 70-12-5, 6-7 cm:

Triquetrorhabdulus carinatus, Discoaster druggii, $D$. nephados, D. dilatus, D. argutus, D. obtusus, D. lidzi. Age: D. druggii Zone (Early Miocene).

\section{HOLE 70A}

Sample 70A-1-2, 6-7 cm:

Sample 70A-1-2, 63-64 cm:

Sample 70A-1-3, 6-7 cm:

Sample 70A-1-4, 6-7 cm:

Sample 70A-1-5, 6-7 cm:

Sample 70A-1-6, 6-7 cm:

Sample 70A-2-1, 6-7 cm:

Sample 70A-2-2, 6-7 cm:

Sample 70A-2-3, 6-7 cm:

Sample 70A-2-4, 6-7 cm:

Sample 70A-2-5, 6-7 cm:

Sample 70A-2-6, 6-7 cm:

Sample 70A-3-5, $12-13 \mathrm{~cm}$

Sample 70A-3-6, Bottom:

Sample 70A-4-2, 6-7 cm:

Sample 70A-4-3, 6-7 cm: 
Sample 70A-4-4, 6-7 cm:

Sample 70A-4-5, 6-7 cm:

Triquetrorhabdulus carinatus, Discoaster nephados, $D$. dilatus, D. argutus, D. obtusus, D. lidzi.

Age: $T$. carinatus Zone (Early Miocene).

Sample 70A-4-6, 6-7 cm:

Flood of coccoliths, very few asteroliths.

Sample 70A-5-4, Bottom:

Sample 70A-5-6, 6-7 cm:

Sample 70A-6-1, 6-7 cm:

Sample 70A-6-2, Bottom:

Sample 70A-6-3, Bottom:

Sphenolithus ciperoensis, Discoaster argutus, D. dilatus, D. nephados, Coccolithus eopelagicus.

Age: Probably $S$. ciperoensis Zone (Late Oligocene).

Sample 70A-6-4, 6-7 cm:

Sample 70A-6-5, Top:

Sample 70A-6-6, 6-7 cm:

Sample 70A-7-2, 1-10 cm:

Sample 70A-7-3, Bottom:

Sample 70A-7-4, 6-7 cm:

Sample 70A-7-5, 6-7 cm:

Sample 70A-8-1, Bottom:

Sample 70A-8-3, Top:

Sample 70A-8-4, 6-7 cm:

Flood of Sphenolithus pacificus, S. ciperoensis, $S$. distentus, Coccolithus eopelagicus, Discoaster nephados, D. dilatus.

Age: $S$. ciperoensis Zone (Late Oligocene).

Sample 70A-8-5, 6-7 cm:

Sample 70A-9-1, Top:

Sample 70A-9-2, 6-7 cm:

Sample 70A-9-4, 6-7 cm:

Sample 70A-9-4, 63-64 cm:

Sample 70A-10-1, 6-7 cm:

Sample 70A-10-2, 6-7 cm:

Sample 70A-10-3, 6-7 cm:

Sample 70A-10-4, 6-7 cm:

Sample 70A-10-5, 6-7 cm:

Sample 70A-11-2, 6-7 cm:

Sample 70A-11-3, 6-7 cm:

Sphenolithus distentus, Discoaster nephados, D. dilatus, Coccolithus eopelagicus, $C$. pelagicus.

Age: Probably Sphenolithus distentus Zone (Late Olig. ocene).

Sample 70A-11-4, 6-7 cm:

Sample 70A-11-5, 6-7 cm:

Sample 70A-12-1, 6-7 cm:

Sample 70A-12-2, 6-7 cm:

Sample 70A-12-3, 6-7 cm:

Sample 70A-12-4, 6-7 cm:

Sphenolithus distentus, $S$. predistentus, and intermediate forms.

Age: Probably S. distentus Zone (Late Oligocene).
Sample 70A-13-2, 6-7 cm:

Very poor assemblage.

Sample 70A-13-3, 6-7 cm:

Sample 70A-13-4, 6-7 cm:

Sample 70A-14-2, 6-7 cm:

Sample 70A-14-3, 6-7 cm:

Sample 70A-15-1, $134 \mathrm{~cm}$ :

Sample 70A-15-2, 6-7 cm:

Sample 70A-15-3, 6-7 cm:

Sample 70A-15-4, 6-7 cm:

Common Sphenolithus predistentus, and many cocco-

liths.

Age: Probably $S$. predistentus Zone (Middle Oligocene).

Sample 70A-16-1, 48-50 cm:

Sample 70A-16-2, 6-7 cm:

Sample 70A-16-3, 10-12 cm:

Sample 70A-17-1, 115-117 cm:

Sample 70A-17-2, 6-7 cm:

Sample 70A-17-3, 6-7 cm:

Sample 70A-17-4, 6-7 cm:

Sample 70A-18-1, 6-7 cm:

Sample 70A-18-2, 6-7 cm:

Sample 70A-19-1, 124-125 cm:

Sample 70A-19-2, 6-7 cm:

Sample 70A-19-3, 6-7 cm:

As above, but with fewer specimens of Sphenolithus predistentus and a flood of $S$. pacificus.

Sample 70A-21-1, $71-72 \mathrm{~cm}$ :

Sample 70A-21-2, 6-7 cm:

Sample 70A-22-2, 6-7 cm:

Sample 70A-23-2, 6-7 cm:

Sample 70A-24-1, 94-95 cm:

Sample 70A-24-2, 6-7 cm:

Sample 70A-24-3, 6-7 cm:

Sample 70A-25-1, 117-118 cm:

Sample 70A-25-2, 6-7 cm:

Sample 70A-25-3, 6-7 cm:

Sample 70A-26-2, ?:

Sample 70A-27-1, 115-116 cm:

Sample 70A-27-2, 6-7 cm:

Coccolithus pelagicus, Reticulofenestra bisecta, Discoaster dilatus, $D$. nephados.

Age: Early Oligocene.

Sample 70A-27-3, 6-7 cm:

Sample 70A-27-3, 6-7 cm:

Sample 70A-28-1, 6-7 cm:

Barren.

\section{HOLE 71}

Sample 71-1-1, 6-7 cm:

Gephyrocapsa oceanica, Cyclococcolithus leptoporus, Ceratolithus cristatus. 
Age: Upper part of the $G$. oceanica Zone (Late Pleistocene).

Sample 71-1-2, 6-7 cm:

Gephyrocapsa oceanica, G. caribbeanica, Pseudoemiliania lacunosa, Ceratolithus cristatus.

Age: Lower part of G. oceanica Zone (Late Pleistocene).

Sample 71-1-3, 6-7 cm

Gephyrocapsa caribbeanica, Pseudoemiliania lacunosa.

Age: G. caribbeanica Zone (Early Pleistocene).

Sample 71-1-4, 6-7 cm:

Sample 71-1-5, 6-7 cm:

Discoaster brouweri, Gephyrocapsa caribbeanica.

Age: D. brouweri Zone (Late Pliocene).

Sample 71-2-2, 6-7 cm:

Discoaster brouweri, D. pentaradiatus, Ceratolithus cristatus.

Age: D. pentaradiatus Zone (Late Pliocene).

Sample 71-2-3, 6-7 cm:

Sample 71-2-4, 6-7 cm:

Discoaster brouweri, D. pentaradiatus, Ceratolithus rugosus.

Age: D. pentaradiatus Zone (Late Pliocene).

Sample 71-2-5, 6-7 cm:

Sample 71-3-1, 6-7 cm:

Sample 71-3-3, 20-21 cm:

Discoaster extensus, D. brouweri, D. pentaradiatus, Ceratolithus rugosus.

Age: D. extensus Zone (Late Pliocene).

Sample 71-3-4, 6-7 cm:

Sample 71-3-5, $24-25 \mathrm{~cm}$ :

Sample 71-3-6, ?:

Discoaster surculus, D. extensus, D. brouweri, Cerato-

lithus rugosus.

Age: D. surculus Zone (Late Pliocene).

Sample 71-4-1, 6-7 cm:

Unusual assemblage consisting almost exclusively of asteroliths listed above, very few coccoliths, no ceratoliths.

Sample 71-4-2, 6-7 cm:

Sample 71-4-3, 10-11 cm:

D. surculus, D. extensus, D. brouweri, Ceratolithus rugosus, $C$. tricorniculatus.

Age: Probably Discoaster asymmetricus Zone (Early Pliocene).

Sample 71-4-4, 6-7 cm:

Sample 71-4-5, 6-7 cm:

Sample 71-5-4, 6-7 cm:

Discoaster asymmetricus, D. surculus, D. extensus,
D. brouweri, Ceratolithus rugosus, C. tricorniculatus. Age: D. asymmetricus Zone (Early Pliocene).

Sample 71-5-5, 6-7 cm:

Sample 71-5-6, 6-7 cm:

Sample 71-6-1, 6-7 cm:

Discoaster surculus, D. extensus, D. brouweri, Ceratolithus tricorniculatus, very few $C$. rugosus.

Age: Probably $C$. rugosus Zone (Early Pliocene).

Sample 71-6-3, 6-7 cm:

Sample 71-6-4, 6-7 cm:

Sample 71-6-5, 6-7 cm:

Discoaster brouweri, D. extensus, D. surculus, many coccoliths.

Age: Probably Ceratolithus tricorniculatus Zone (Late Miocene).

Sample 71-7-1, 6-7 cm:

Sample 71-7-2, $6 \mathrm{~cm}$ :

Sample 71-7-3, $6 \mathrm{~cm}$ :

Sample 71-7-4, $6 \mathrm{~cm}$ :

Sample 71-7-5, $6 \mathrm{~cm}$ :

Discoaster quinqueramus, D. brouweri, D. extensus, many coccoliths.

Age: D. quinqueramus Zone (Late Miocene).

Sample 71-7-6, $6 \mathrm{~cm}$ :

Discoaster challengeri, $D$. cf. hamatus, $D$. brouweri, $D$. extensus.

Age: Late Miocene.

Sample 71-8-1, 6-7 cm:

Sample 71-8-2, 15-16 cm:

Sample 71-8-5, $15-16 \mathrm{~cm}$ :

Sample 71-9-1, 6-7 cm:

Sample 71-9-2, $6 \mathrm{~cm}$ :

Discoaster hamatus, D. challengeri, D. brouweri, D. extensus, many coccoliths.

Age: D. hamatus Zone (Late Miocene).

Sample 71-9-3, $6 \mathrm{~cm}$ :

Many five-rayed specimens of Discoaster brouweri.

Age: Late Miocene.

Sample 71-10-1, $6 \mathrm{~cm}$ :

Sample 71-10-2, $6 \mathrm{~cm}$ :

Sample 71-10-3, $6 \mathrm{~cm}$ :

Sample 71-10-4, $6 \mathrm{~cm}$ :

Sample 71-10-5, $6 \mathrm{~cm}$ :

Sample 71-10-6, $6 \mathrm{~cm}$ :

Unusual assemblages with many coccoliths and few asteroliths; asteroliths frequently overgrown with calcite. Discoaster brouweri, D. extensus, D. kugleri. Age: D. kugleri Zone (Middle Miocene).

Sample 71-11-3, $6 \mathrm{~cm}$ :

Sample 71-11-5, $6 \mathrm{~cm}$ : 
Sample 71-12-1, $6 \mathrm{~cm}$ :

Sample 71-12-2, $6 \mathrm{~cm}$ :

Discoaster cf. exilis, D. extensus, many coccoliths, but few and poorly preserved asteroliths.

Age: Probably D. exilis Zone (Middle Miocene).

Sample 71-12-4, $6 \mathrm{~cm}$ :

Sample 71-12-5, $6 \mathrm{~cm}$ :

Sample 71-13-1, $6 \mathrm{~cm}$ :

Sample 71-13-3, $6 \mathrm{~cm}$ :

Sample 71-14-1, $6 \mathrm{~cm}$ :

Sample 71-14-3, $6 \mathrm{~cm}$ :

Sample 71-14-5, $6 \mathrm{~cm}$ :

Sample 71-15-1, $6 \mathrm{~cm}$ :

Sample 71-15-5, $6 \mathrm{~cm}$ :

Discoaster cf. trinidadensis, D. nephados; assemblages with many coccoliths, but few and poorly preserved asteroliths.

Sample 71-16-1, 70-75 cm:

Discoaster brouweri, D. extensus, and other forms from higher in section.

Sample 71-16-2, 71-72 cm:

Sample 71-17-3, $6 \mathrm{~cm}$ :

Discoaster brouweri, Sphenolithus heteromorphus, Discoaster deflandrei, D. extensus, Coccolithus pelagicus Mixed assemblage of S. heteromorphus Zone and higher (Middle Miocene).

Sample 71-17-4, $6 \mathrm{~cm}$ :

Sample 71-17-5, $6 \mathrm{~cm}$ :

Discoaster deflandrei, D. nephados, D. argutus, rare Sphenolithus heteromorphus; probably $S$. heteromorphus Zone (Middle Miocene).

Sample 71-17-6, $13 \mathrm{~cm}$ :

Sample 71-18-2, $6 \mathrm{~cm}$ :

Sample 71-18-3, $6 \mathrm{~cm}$ :

Sample 71-18-4, $6 \mathrm{~cm}$ :

Sample 71-18-5, $6 \mathrm{~cm}$ :

Discoaster deflandrei, D. nephados, D. lidzi, D. argutus, Sphenolithus heteromorphus; $S$. heteromorphus Zone (Middle Miocene).

Sample 71-19-1, $6 \mathrm{~cm}$ :

Sample 71-19-2, $6 \mathrm{~cm}$ :

Sample 71-19-5, $6 \mathrm{~cm}$ :

Sample 71-20-2, $6 \mathrm{~cm}$ :

Sample 71-20-4, $6 \mathrm{~cm}$ :

Sample 71-20-6, $6 \mathrm{~cm}$ :

Sample 71-21-2, $71 \mathrm{~cm}$ :

Sample 71-21-4, $6 \mathrm{~cm}$ :

Sample 71-21-6, $6 \mathrm{~cm}$ :

Sample 71-22-4, $6 \mathrm{~cm}$ :

Sample 71-22-6, $6 \mathrm{~cm}$ :

Sample 71-23-3, $6 \mathrm{~cm}$ :
Sample 71-23-4, $6 \mathrm{~cm}$ :

Sample 71-23-6, $6 \mathrm{~cm}$ :

Sample 71-24-3, $6 \mathrm{~cm}$ :

Sample 71-24-4, $6 \mathrm{~cm}$ :

Sample 71-25-3, 9-10 cm:

Sample 71-25-5, $6 \mathrm{~cm}$ :

Discoaster deflandrei, D. aulakos, D. nephados, D. cf. lidzi, D. argutus, Sphenolithus heteromorphus; $S$. heteromorphus Zone (Middle Miocene).

Sample 71-26-2, $6 \mathrm{~cm}$ :

Sample 71-26-4, $6 \mathrm{~cm}$ :

Sample 71-26-6, $6 \mathrm{~cm}$ :

Sample 71-28-2, $6 \mathrm{~cm}$ :

Sample 71-29-1, $6 \mathrm{~cm}$ :

Sample 71-29-3, $6 \mathrm{~cm}$ :

Sample 71-29-5, $6 \mathrm{~cm}$ :

Sample 71-29-6, $6 \mathrm{~cm}$ :

Sample 71-30-2, $6 \mathrm{~cm}$ :

Sample 71-30-4, $6 \mathrm{~cm}$ :

Discoaster lidzi, D. deflandrei, D. nephados, Sphenolithus belemnos.

Age: S. belemnos Zone (Early Miocene).

Note: Helicopontosphaera does not occur in these samples so that recognition of the $H$. ampliaperta Zone is impossible.

Sample 71-30-6, $6 \mathrm{~cm}$ :

Sample 71-31-2, $6 \mathrm{~cm}$ :

Sample 71-31-4, $6 \mathrm{~cm}$ :

Sample 71-31-6, $6 \mathrm{~cm}$ :

Sample 71-32-2, $6 \mathrm{~cm}$ :

Sample 71-32-4, $6 \mathrm{~cm}$ :

Sample 71-32-6, $6 \mathrm{~cm}$ :

Sample 71-33-5, $6 \mathrm{~cm}$ :

Sample 71-33-6, $43 \mathrm{~cm}$ :

Sample 71-34-2, $6 \mathrm{~cm}$ :

Sample 71-34-4, $6 \mathrm{~cm}$ :

Sample 71-34-6, $6 \mathrm{~cm}$ :

Sample 71-35-2, $6 \mathrm{~cm}$ :

Sample 71-35-4, $6 \mathrm{~cm}$ :

Sample 71-35-6, $6 \mathrm{~cm}$ :

Assemblages almost entirely coccoliths, few asteroliths and no sphenoliths present.

Age: Probably Sphenolithus belemnos Zone, based on position above the highest occurrence of Triquetrorhabdulus carinatus (Early Miocene).

Sample 71-36-4, $6 \mathrm{~cm}$ :

Sample 71-36-6, $6 \mathrm{~cm}$ :

Sample 71-38-4, $6 \mathrm{~cm}$ :

Sample 71-38-6, $6 \mathrm{~cm}$ :

Sample 71-40-3, $10 \mathrm{~cm}$ :

Sample 71-40-5, $10 \mathrm{~cm}$ :

Sample 71-42-1, $6 \mathrm{~cm}$ :

Sample 71-42-4, $6 \mathrm{~cm}$ :

Sample 71-42-5, $6 \mathrm{~cm}$ : 
Sample 71-42-6, $6 \mathrm{~cm}$ :

Sample 71-43-2, $6 \mathrm{~cm}$ :

Sample 71-43-3, $6 \mathrm{~cm}$ :

Sample 71-44-1, $6 \mathrm{~cm}$ :

Sample 71-44-3, $6 \mathrm{~cm}$ :

Sample 71-44-4, $6 \mathrm{~cm}$ :

Sample 71-44-6, $6 \mathrm{~cm}$ :

Triquetrorhabdulus carinatus, Discoaster deflandrei, $D$. nephados, many coccoliths.

Age: Probably $T$. carinatus Zone, Early Miocene.

\section{HOLE 72}

Sample 72-1-1, $6 \mathrm{~cm}$ :

Gephyrocapsa oceanica, Cyclococcolithus leptoporus, Helicopontosphaera kamptneri, Ceratolithus cristatus. Age: Upper part of the G. oceanica Zone, (Late Pleistocene).

Sample 72-1-2, $6 \mathrm{~cm}$ :

Sample 72-1-3, $6 \mathrm{~cm}$ :

Gephyrocapsa oceanica, Pseudoemiliania lacunosa, Ceratolithus cristatus.

Age: Lower part of the $G$. oceanica Zone, (Late Pleistocene).

Sample 72-1-5, $6 \mathrm{~cm}$ :

Sample 72-1-6, $6 \mathrm{~cm}$ :

Gephyrocapsa caribbeanica, Pseudoemiliania lacunosa.

Age: G. caribbeanica Zone (Early Pleistocene).

Sample 72-2-1, $116 \mathrm{~cm}$ :

Sample 72-2-2, $6 \mathrm{~cm}$ :

Sample 72-2-3, $16 \mathrm{~cm}$ :

Discoaster brouweri, D. calcaris, D. pentaradiatus, Ceratolithus rugosus.

Age: D. pentaradiatus Zone (Late Pliocene).

Sample 72-2-4, $6 \mathrm{~cm}$ :

Sample 72-2-5, $6 \mathrm{~cm}$ :

Discoaster extensus, D. brouweri, D. pentaradiatus, $D$. cf. surculus.

Age: D. extensus Zone (Late Pliocene).

Sample 72-3-1, $6 \mathrm{~cm}$ :

Discoaster surculus, D. extensus, $D$. brouweri.

Age: D. surculus Zone (Late Pliocene).

Sample 72-3-2, $6 \mathrm{~cm}$ :

Sample 72-3-3, $6 \mathrm{~cm}$ :

Sample 72-3-4, $6 \mathrm{~cm}$ :

Sample 72-3-5, $6 \mathrm{~cm}$ :

Poor assemblages with Discoaster brouweri, D. extensus, and coccoliths.

Age: Late Miocene or Early Pliocene.

Sample 72-4-1, $6 \mathrm{~cm}$ :

Discoaster extensus, D. dilatus, D. brouweri, D. kugleri. Age: Probably D. kugleri Zone (Middle Miocene).
Sample 72-4-2, $6 \mathrm{~cm}$ :

Sample 72-4-3, $6 \mathrm{~cm}$ :

Sample 72-4-4, $6 \mathrm{~cm}$ :

Assemblages with heavily calcified asteroliths; exact age assignment impossible.

Sample 72-4-5, $6 \mathrm{~cm}$ :

Discoaster argutus, D. extensus, D. dilatus, D. nephados. Age: Early Miocene.

Sample 72-5-1, $6 \mathrm{~cm}$ :

Discoaster extensus, D. nephados, D. dilatus, Sphenolithus belemnos.

Age: S. belemnos Zone (Early Miocene).

Sample 72-5-5, $6 \mathrm{~cm}$ :

Discoaster quinqueramus, D. brouweri, D. variabilis, $D$. extensus, D. nephados; mixed assemblage from higher in hole.

Sample 72-5-6, $6 \mathrm{~cm}$ :

Discoaster brouweri, D. nephados, D. dilatus, D. extensus, Sphenolithus distentus.

Age: Probably Early Miocene, with admixed material from higher in hole.

Sample 72-6-2, $6 \mathrm{~cm}$ :

Sample 72-6-4, $6 \mathrm{~cm}$ :

Sample 72-6-6, $6 \mathrm{~cm}$ :

Triquetrorhabdulus carinatus, Discoaster lidzi, D. dilatus, D. extensus, D. nephados.

Age: T. carinatus Zone (Early Miocene).

Sample 72-7-1, $6 \mathrm{~cm}$ :

Sample 72-7-2, $6 \mathrm{~cm}$ :

Sample 72-7-3, $6 \mathrm{~cm}$ :

Sample 72-7-4, $6 \mathrm{~cm}$ :

Sample 72-7-5, $6 \mathrm{~cm}$ :

Sample 72-7-6, $6 \mathrm{~cm}$ :

Discoaster lidzi, D. nephados, Sphenolithus ciperoensis. Age: Probably Sphenolithus distentus Zone (Late Oligocene).

Sample 72-8-5, $6 \mathrm{~cm}$ :

Sample 72-8-6, 6-7 cm:

Sphenolithus predistentus, few asteroliths, many small coccoliths.

Age: Probably $S$. predistentus Zone (Middle Oligocene).

Sample 72-9-2, $6 \mathrm{~cm}$ :

Sample 72-9-3, $6 \mathrm{~cm}$ :

Sample 72-9-4, $6 \mathrm{~cm}$ :

Sample 72-9-5, $6 \mathrm{~cm}$ :

Sample 72-9-6, $6 \mathrm{~cm}$ :

Reticulofenestra bisecta, R. umbilica, many small coccoliths, few asteroliths.

Age: Early Oligocene. 
Sample 72-10-3, $4 \mathrm{~cm}$ :

Sample 72-10-5, 120-135 cm:

Sample 72-11-1, 22-23 cm:

Discoaster barbadiensis, D. saipanensis, D. tani, Reticulofenestra umbilica.

Age: Late Eocene.

\section{HOLE 72A}

Sample 72A-1-1, $110 \mathrm{~cm}$ :

Sample 72A-1-2, $6 \mathrm{~cm}$ :

Sample 72A-1-3, $6 \mathrm{~cm}$ :

Sample 72A-1-4, $46 \mathrm{~cm}$ :

Sample 72A-2-1, $6 \mathrm{~cm}$ :

Sample 72A-2-2, $6 \mathrm{~cm}$ :

Sample 72A-2-3, $6 \mathrm{~cm}$ :

Gephyrocapsa caribbeanica, Pseudoemiliania lacunosa, Cyclococcolithus leptoporus, Helicopontosphaera kamptneri, Ceratolithus cristatus.

Age: Gephyrocapsa caribbeanica Zone (Early Pleistocene).

Sample 72A-2-4, $6 \mathrm{~cm}$ :

Sample 72A-2-5, $6 \mathrm{~cm}$ :

Sample 72A-3-2, $6 \mathrm{~cm}$ :

Sample 72A-3-3, $6 \mathrm{~cm}$ :

Sample 72A-3-4, $6 \mathrm{~cm}$ :

Sample 72A-3-5, $6 \mathrm{~cm}$ :

Sample 72A-4-2, $6 \mathrm{~cm}$ :

Sample 72A-4-3, $6 \mathrm{~cm}$ :

Sample 72A-4-6, $6 \mathrm{~cm}$ :

Discoaster brouweri calcaris, Coccolithus pelagicus.

Age: D. brouweri Zone (Late Pliocene).

Sample 72A-5-2, $6 \mathrm{~cm}$ :

Sample 72A-5-3, $6 \mathrm{~cm}$ :

Discoaster pentaradiatus, D. brouweri.

Age: D. pentaradiatus Zone (Late Pliocene).

Sample 72A-5-4, $6 \mathrm{~cm}$ :

Discoaster extensus, D. pentaradiatus, D. brouweri, Ceratolithus rugosus, $C$. tricorniculatus.

Age: D. extensus Zone (Late Pliocene).

Sample 72A-5-5, $6 \mathrm{~cm}$ :

Sample 72A-5-6, $6 \mathrm{~cm}$ :

Sample 72A-6-1, $6 \mathrm{~cm}$ :

Sample 72A-6-2, $6 \mathrm{~cm}$ :

Sample 72A-6-3, $6 \mathrm{~cm}$ :

Discoaster surculus, D. extensus, D. brouweri, Cerato-

lithus rugosus, $C$. tricorniculatus.

Age: D. surculus Zone (Late Pliocene).

Sample 72A-6-4, $6 \mathrm{~cm}$ :

Sample 72A-6-5, $6 \mathrm{~cm}$ :

As above, but heavily calcified.

Sample 72A-6-6, $6 \mathrm{~cm}$ :

Reticulofenestra pseudoumbilica, Ceratolithus rugosus,
C. tricorniculatus.

Age: $R$. pseudoumbilica Zone (Late Pliocene).

\section{HOLE 73}

Sample 73-1-1, $6 \mathrm{~cm}$ :

Sample 73-1-2, $6 \mathrm{~cm}$ :

Sample 73-2-1, $6 \mathrm{~cm}$ :

Gephyrocapsa oceanica, Cyclococcolithus leptoporus, Ceratolithus cristatus, Helicopontosphaera kamptneri. Age: Upper part of the G. oceanica Zone (Late Pleistocene).

Sample 73-2-2, $6 \mathrm{~cm}$ :

Sample 73-2-3, $6 \mathrm{~cm}$ :

Sample 73-2-4, $6 \mathrm{~cm}$ :

Sample 73-2-5, $6 \mathrm{~cm}$ :

Gephyrocapsa oceanica, G. caribbeanica, Cyclococcolithus leptoporus, Ceratolithus cristatus, Pseudoemiliania lacunosa.

Age: Lower part of the G. oceanica Zone (Late Pleistocene).

Sample 73-3-1, $96 \mathrm{~cm}$ :

Sample 73-3-2, $6 \mathrm{~cm}$ :

Sample 73-3-3, $6 \mathrm{~cm}$ :

Sample 73-3-4, $6 \mathrm{~cm}$ :

Sample 73-3-5, $6 \mathrm{~cm}$ :

Sample 73-3-6, $6 \mathrm{~cm}$ :

Gephyrocapsa caribbeanica, Pseudoemiliania lacunosa, Cyclococcolithus leptoporus.

Age: G. caribbeanica Zone (Early Pleistocene).

Sample 73-4-1, $6 \mathrm{~cm}$ :

Sample 73-4-2, $6 \mathrm{~cm}$ :

Sample 73-4-3, $6 \mathrm{~cm}$ :

Sample 73-4-4, $6 \mathrm{~cm}$ :

Discoaster brouweri, Gephyrocapsa caribbeanica,

Pseudoemiliania lacunosa.

Age: D. brouweri Zone (Late Pliocene).

Sample 73-4-5, $6 \mathrm{~cm}$ :

Sample 73-4-6, $6 \mathrm{~cm}$ :

Sample 73-5-2, $7 \mathrm{~cm}$ :

Sample 73-5-5, 6-7 cm:

Sample 73-5-6, 6-7 cm:

Sample 73-6-1, 6-7 cm:

Discoaster pentaradiatus, D. brouweri.

Age: D. pentaradiatus Zone (Late Pliocene).

Sample 73-6-2, 6-7 cm:

Discoaster cf. extensus, D. pentaradiatus, D. brouweri; D. extensus Zone (Late Pliocene).

Sample 73-6-3, 6-7 cm:

Sample 73-6-4, 6-7 cm:

Sample 73-6-5, 6-7 cm:

Sample 73-6-6, 6-7 cm:

Discoaster cf. variabilis, D. extensus, D. pentaradiatus, 
D. brouweri, very few coccoliths.

Age: D. extensus Zone (Late Pliocene).

Sample 73-7-1, 6-7 cm:

Sample 73-7-3, 6-7 cm:

Sample 73-7-4, 6-7 cm:

Sample 73-7-5, 6-7 cm:

Discoaster cf. surculus, D. extensus, D. brouweri, Ceratolithus rugosus.

Age: Lowermost $D$. extensus Zone or uppermost $D$. surculus Zone (Late Pliocene).

Sample 73-7-6, 6-7 cm:

Sample contains abundant Ceratolithus tricorniculatus, but no $C$. rugosus.

Sample 73-8-1, 6-7 cm:

Sample 73-8-2, 6-7 cm:

Sample 73-8-4, 6-7 cm:

Sample 73-8-6, 6-7 cm:

Sample 73-9-2, $6 \mathrm{~cm}$ :

Discoaster surculus, D. extensus, D. brouweri, Ceratolithus tricorniculatus.

Age: Late Miocene or Early Pliocene.

Sample 73-9-6, $6 \mathrm{~cm}$ :

Reticulofenestra pseudoumbilica, Discoaster druggii, $D$. dilatus, D. brouweri, D. extensus; mixed Miocene assemblage.

Sample 73-10-2, $6 \mathrm{~cm}$ :

Sample 73-10-4, $6 \mathrm{~cm}$ :

Sample 73-10-6, $6 \mathrm{~cm}$ :

Discoaster cf. druggii, D. dilatus, D. nephados, D. extensus.

Age: Early Miocene.

Sample 73-11-2, $6 \mathrm{~cm}$ :

Sample 73-11-4, $6 \mathrm{~cm}$ :

Sample 73-11-6, $6 \mathrm{~cm}$ :

Discoaster nephados, D. dilatus, Sphenolithus heteromorphus?

Age: Probably Helicopontosphaera ampliaperta Zone (Early Miocene).

Sample 73-12-2, $6 \mathrm{~cm}$ :

Discoaster nephados, D. dilatus, Sphenolithus belemnos. Age: Probably $S$. belemnos Zone (Early Miocene).

Sample 73-12-4, $6 \mathrm{~cm}$ :

Sample 73-12-6, $6 \mathrm{~cm}$ :

Triquetrorhabdulus carinatus, Discoaster dilatus, D. nephados.

Age: $T$. carinatus Zone (Early Miocene).

Sample 73-13-2, $6 \mathrm{~cm}$ :

Triquetrorhabdulus carinatus, Discoaster dilatus, D. nephados, Sphenolithus distentus.
Age: Lowermost $T$. carinatus Zone or uppermost Sphenolithus ciperoensis Zone (Early Miocene or Late Oligocene).

Sample 73-13-4, 6-7 cm:

Sample 73-13-6, $6 \mathrm{~cm}$ :

Sample 73-14-2, $6 \mathrm{~cm}$ :

Sphenolithus predistentus, with nondescript asteroliths and many small coccoliths.

Age: S. predistentus Zone (Middle Oligocene).

Sample 73-14-3, $6 \mathrm{~cm}$ :

Sphenolithus predistentus, Discoaster tani ornatus, flood of small coccoliths and sphenoliths.

Age: S. predistentus Zone (Middle Oligocene).

Sample 73-14-4, $6 \mathrm{~cm}$ :

Sample 73-14-6, $6 \mathrm{~cm}$ :

Sample 73-15-2, 6-7 cm:

Sample 73-15-4, $6 \mathrm{~cm}$ :

Sample 73-15-6, $6 \mathrm{~cm}$ :

Sample 73-16-2, 6-7 cm:

Sample 73-16-4, $6 \mathrm{~cm}$ :

Sample 73-16-6, $6 \mathrm{~cm}$ :

Sample 73-17-2, 6-7 cm:

Sample 73-17-4, 6-7 cm:

Sample 73-17-6, 6-7 cm:

Flood of Sphenolithus pacificus and many coccoliths.

Age: Early Oligocene.

Sample 73-18-6, $16 \mathrm{~cm}$ :

Sample 73-19-2, $6 \mathrm{~cm}$ :

Many Reticulofenestra bisecta, $R$. umbilica.

Age: Early Oligocene.

Sample 73-19-4, $6 \mathrm{~cm}$ :

Very poor assemblage, with rims of Reticulofenestra umbilica only.

Sample 73-20-4, $6 \mathrm{~cm}$ :

Discoaster barbadiensis, D. saipanensis, Reticulofenestra umbilica.

Age: Late Eocene.

Sample 73-21-2, $6 \mathrm{~cm}$ :

Discoaster nephados, D. extensus, D. barbadiensis, D. saipanensis.

Age: Mixed Miocene and Eocene.

\section{HOLE 74}

Sample 74-1-2, $6 \mathrm{~cm}$ :

Barren.

Sample 74-3-4, $6 \mathrm{~cm}$ :

Sample 74-4-2, $6 \mathrm{~cm}$ :

Sample 74-4-4, $6 \mathrm{~cm}$ : 
Sample 74-5-2, $6 \mathrm{~cm}$ :

Triquetrorhabdulus carinatus, Discoaster nephados, $D$. extensus, D. dilatus.

Age: T. carinatus Zone (Early Miocene).

Sample 74-5-4, $6 \mathrm{~cm}$ :

Sample 74-5-6, $6 \mathrm{~cm}$ :

Sample 74-6-2, $6 \mathrm{~cm}$ :

Sample 74-6-4, $6 \mathrm{~cm}$ :

Sample 74-6-6, $6 \mathrm{~cm}$ :

Sample 74-7-2, $6 \mathrm{~cm}$ :

Triquetrorhabdulus carinatus, Discoaster nepinados, $D$. dilatus, D. cf. deflandrei, Sphenolithus ciperoensis.

Age: $T$. carinatus Zone (Early Miocene) or $S$. ciperoensis Zone (Late Oligocene).

Sample 74-7-4, $6 \mathrm{~cm}$ :

Sample 74-7-5, 6-7 cm:

Triquetrorhabdulus carinatus, Sphenolithus ciperoensis, S. distentus.

Age: Lowermost $S$. ciperoensis Zone or uppermost $S$. distentus Zone (Late Oligocene).

Sample 74-8-2, 6-7 cm:

Flood of Sphenolithus pacificus.

Sample 74-8-3, 6-7 cm:

Sphenolithus predistentus, Discoaster deflandrei, many coccoliths.

Age: S. predistentus Zone (Middle Oligocene).

Sample 74-9-1, 63-64 cm:

Sample 74-9-2, 6-7 cm:

Sample 74-9-4, 6-7 cm:

Sphenolithus predistentus, Discoaster tani ornatus, many coccoliths.

Age: S. predistentus Zone (Middle Oligocene).

Sample 74-9-5, 6-7 cm:

Sample 74-10-1, 12-13 cm:

Sample 74-11-1, $100 \mathrm{~cm}$ :

Very abundant Sphenolithus pacificus, $S$. predistentus, Discoaster cf. tani ornatus, Reticulofenestra bisecta.

Age: Probably S. predistentus Zone (Middle Oligocene).

Sample 74-12-3, $9 \mathrm{~cm}$ :

Sphenolithus belemnos??; sample rich in organic matter, with few poorly preserved coccoliths and sphenoliths, probably from higher in hole.

\section{HOLE 75}

Sample 75-1-4, $6 \mathrm{~cm}$ :

Sample 75-2-2, $6 \mathrm{~cm}$ :

Sample $75-2-5,6 \mathrm{~cm}$ :

Discoaster nephados, $D$. cf. druggii, $D$. dilatus, $D$. adamanteus, Coronocyclus nitescens.

Age: Early Miocene.

Sample 75-3-3, $6 \mathrm{~cm}$ :

Sample 75-3-5, $6 \mathrm{~cm}$ :

Sample 75-4-2, 6-7 cm:

Sample 75-5-2, 6-7 cm:

Triquetrorhabdulus carinatus, Discoaster nephados, $D$. cf. druggii, $D$. dilatus.

Age: Lowermost $D$. druggii Zone or uppermost $T$. carinatus Zone (Early Miocene).

Sample 75-5-5, 6-7 cm:

Triquetrorhabdulus carinatus, Sphenolithus ciperoensis, Discoaster nephados.

Age: $T$. carinatus Zone or S. ciperoensis Zone (Early Miocene or Late Oligocene).

Sample 75-8-3, 6-7 cm:

Sample 75-8-5, 6-7 cm:

Sample 75-9-5, 6-7 cm:

Sphenolithus predistentus, Discoaster tani ornatus, Coccolithus eopelagicus, Cyclococcolithus floridanus. Age: S. predistentus Zone (Middle Oligocene).

\section{REFERENCES}

Boudreaux, J. E. and Hay, W. W., 1969. Calcareous nannoplankton and biostratigraphy of the Late Pliocene-Pleistocene-Recent sediments in the Submarex cores. Rev. Micropaleont. 1.

Bramlette, M. N. and Wilcoxon, J. A., 1967. Middle Tertiary calcareous nannoplankton of the Cipero section, Trinidad, W. I., Tulane Studies Geol. 5, 93.

Hay, W. W., 1970. Preliminary dating by fossil calcareous nannoplankton. In Bader, R. G. et al., 1970, Initial Reports of the Deep Sea Drilling Project, Volume IV. Washington (U. S. Government Printing Office).

Hay, W. W., Mohler, H. P., Roth, P. H., Schmidt, R. R. and Boudreaux, J. E., 1967. Calcareous nannoplankton zonation of the Cenozoic of the Gulf Coast and Caribbean-Antillean area and transoceanic correlation. Trans. Gulf Coast Assoc. Geol. Soc. 17, 428.

Martini, E. and Worsley, T., 1970. Standard Neogene calcareous nannoplankton zonation. Nature 225, 289. 